\title{
Gallium-67 myocardial imaging for the detection of myocarditis in the acute phase of Kawasaki disease (mucocutaneous lymph node syndrome): the usefulness of single photon emission computed
} tomography

\author{
HIROYUKI MATSUURA, TAKASHI ISHIKITA, SHIN YAMAMOTO, \\ TETSUROW UMEZAWA, RYUICHI ITO, REIKO HASHIGUCHI, \\ TSUTOMU SAJI, NORIO MATSUO, MASAAKI TAKANO*
}

From the Department of Paediatrics, Toho University, School of Medicine; and the ${ }^{\star}$ Department of Radiology, Omori Hospital, Toho University, Ohta-ku, Tokyo, Japan

SUMMARY Myocardial imaging with gallium-67 citrate was used to detect myocarditis in 46 consecutive infants and children ( 31 boys and 15 girls, mean age 21 months) with Kawasaki disease. In all of them planar imaging (group A) was performed at 6 hours and at 48 or 72 hours after the intravenous administration of a mean (SD) dose of gallium-67 citrate $(0.07(0.02)$ $\mathrm{mCi} / \mathrm{kg}$ ). Thirty four patients ( 24 boys and 10 girls, mean age 21 months) also had single photon emission computed tomography imaging (group B) soon after planar imaging. The patients had been ill for from 5 days to 16 days (mean (SD) 10.5 (2.4) days in group A and 10.6 (3.0) days in group B). The colour images obtained at 48 or 72 hours were positive in $41 \%$ of group $A$ and in $64 \%$ of group B. Among the patients with clinically suspected myocarditis, $63 \%$ in group A and $80 \%$ in group B had positive myocardial images. Single photon emission computed tomography imaging permitted the identification of tracer in the myocardium, the pericardium only, or in the heart chambers.

Myocardial imaging with gallium-67 citrate, especially when used with single photon emission computed tomography imaging, is useful for the detection of myocarditis in the acute phase of Kawasaki disease.

Kawasaki disease (mucocutaneous lymph node syndrome) is a disease of unknown aetiology and pathogenesis that usually affects infants and children under four years of age. About $10-20 \%$ of these patients have coronary aneurysms that predispose them to sudden death from acute myocardial infarction. Most patients also have cardiovascular signs such as gallop rhythm and distant heart sounds, electrocardiographic abnormalities, and

Requests for reprints to Dr Hiroyuki Matsuura, Department of Paediatrics, Toho University School of Medicine, 6-11-1 Omorinishi, Ohta-ku, Tokyo, Japan 143.

Accepted for publication 27 April 1987 pericardial effusion; these are thought to be caused by myocarditis, pericarditis, and endocarditis. ${ }^{12}$ Some histopathological studies on necropsy cases and series of endomyocardial biopsy specimens have confirmed that myocarditis is often present in the acute phase of Kawasaki disease. Because treatment of myocarditis may improve the prognosis, new diagnostic techniques are needed to detect this abnormality in infants and children with Kawasaki disease. We have evaluated the usefulness of myocardial imaging with gallium-67 citrate (especially when this radionuclide is used for single photon emission computed tomography) in the detection of myocarditis in patients with acute phase Kawasaki disease. 
Patients-We examined 46 consecutive patients with Kawasaki disease ( 31 boys and 15 girls, age range 6 months to 5 years and 11 months, mean 1 year and 9 months) who were admitted to the Toho University hospital from July 1985 to March 1986. In all of these patients clinical diagnosis was based on the criteria set by the Mucocutaneous Lymph Node Syndrome Research Committee of the Ministry of Health and Welfare of Japan in $1984 .^{3}$

Methods-In the acute phase (the illness had lasted from 5 days to 16 days; mean (SD) 10.5 (2.4) days) $0.5-1.0 \mathrm{mCi}$ of gallium-67 citrate (mean (SD) 0.07 $(0.02) \mathrm{mCi} / \mathrm{kg}$ ) was administered intravenously; the amount administered depended on the child's body weight. Planar imaging was performed in the anterior and in the $45^{\circ}$ left anterior oblique projections in all 46 patients (group A) 6 hours and 48 or 72 hours after injection of the tracer. Soon after planar imaging 34 patients were also examined by single photon emission computed tomography imaging (group B); the gamma camera was rotated around the patient from the $40^{\circ}$ right anterior to the $50^{\circ}$ left posterior projections. These $180^{\circ}$ were divided into 32 equal angles; 6 hours after injection of the tracer $30 \mathrm{sec}-$ onds per angle and 48 or 72 hours afterwards 50 seconds per angle were required for imaging. Both planar and single photon emission computed tomography imaging were performed with a ZLC 7500 gamma camera, Shimazu Inc., using medium energy, high sensitivity collimator. The data obtained were recorded and analysed to make colour images by computer (Scintipac 2400 , Shimazu Inc.).

Three paediatric cardiologists classified the colour images of the anterior view in planar imaging and of the transverse plane view against the truncal axis in single photon emission computed tomography imaging at 48 or 72 hours into four grades. Each was unaware of the clinical data of the patients and of the judgements made by the other two.

The four grades were: negative (-), heart not identified; positive $1+$, showing a vague silhouette of the heart in planar imaging or a vague or incomplete outline of the ventricular walls by single photon emission computed tomography imaging

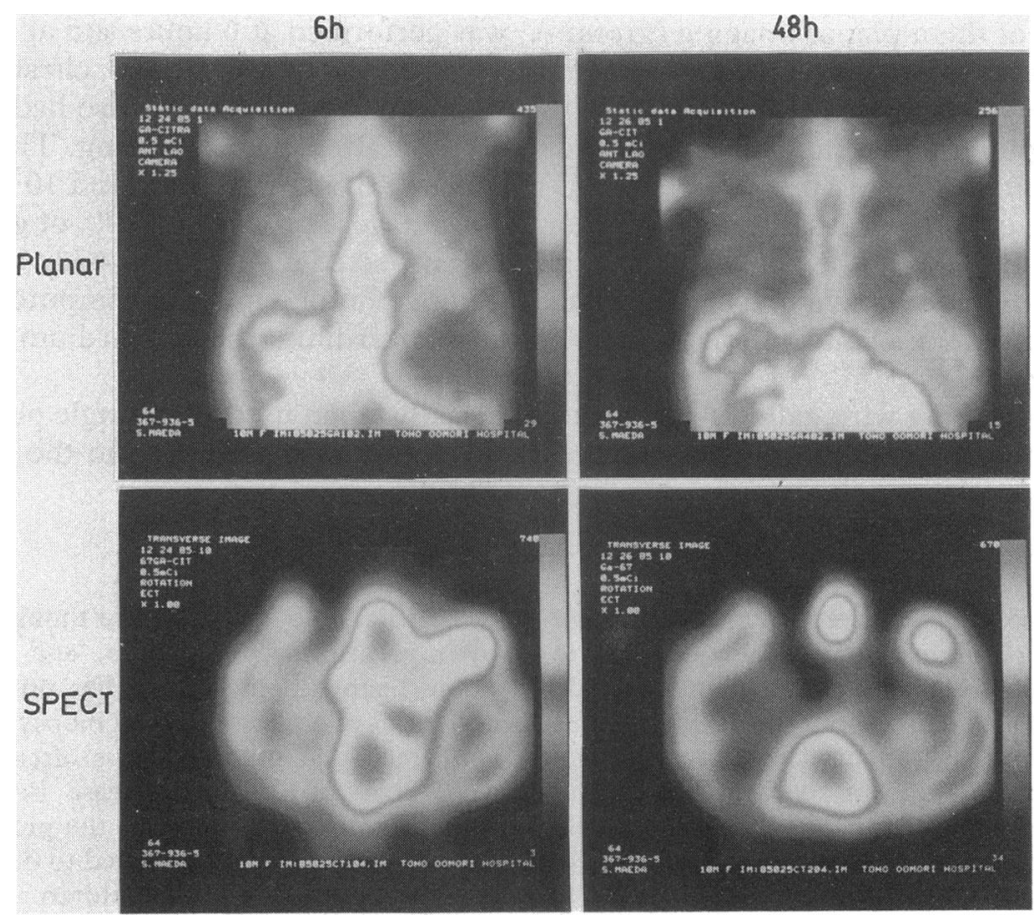

Fig 1 Planar and single photon emission computed tomographic (SPECT) images of a typical negative case. There was a remarkable accumulation of gallium-67 (see cardiac silhouette) in the planar image at 6 hours (top left). Gallium-67 was localised both in the ventricular cavity and in the myocardium in the SPECT image (bottom left). No accumulation of gallium-67 in the heart was seen in planar or SPECT images at 48 hours (top right and bottom right). 

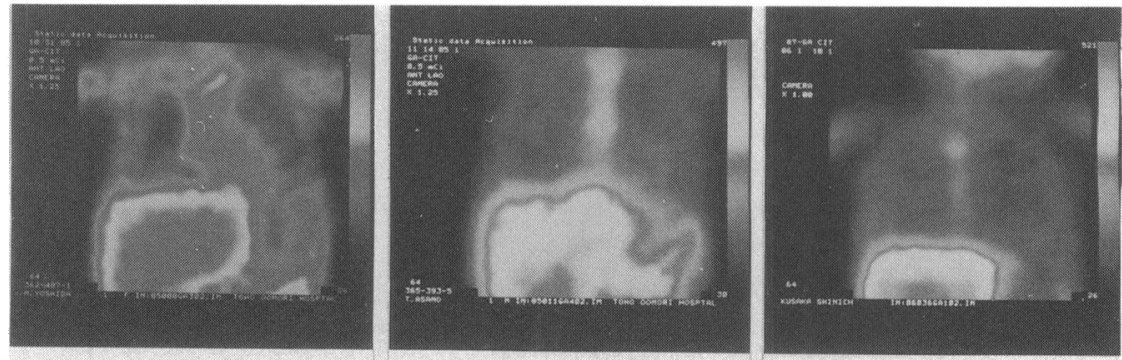

(a)
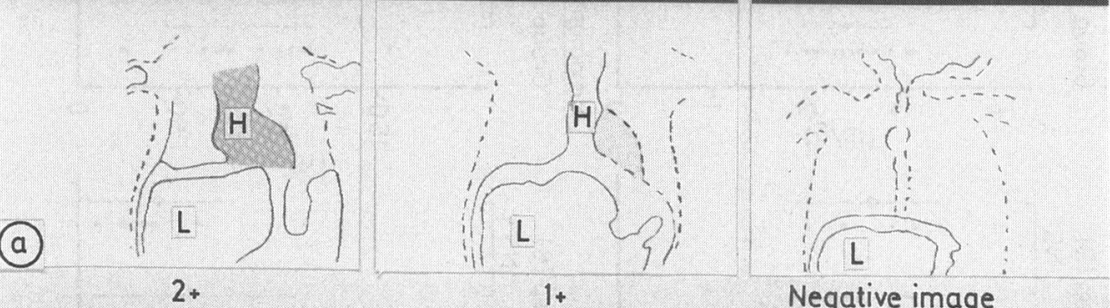

Negative image
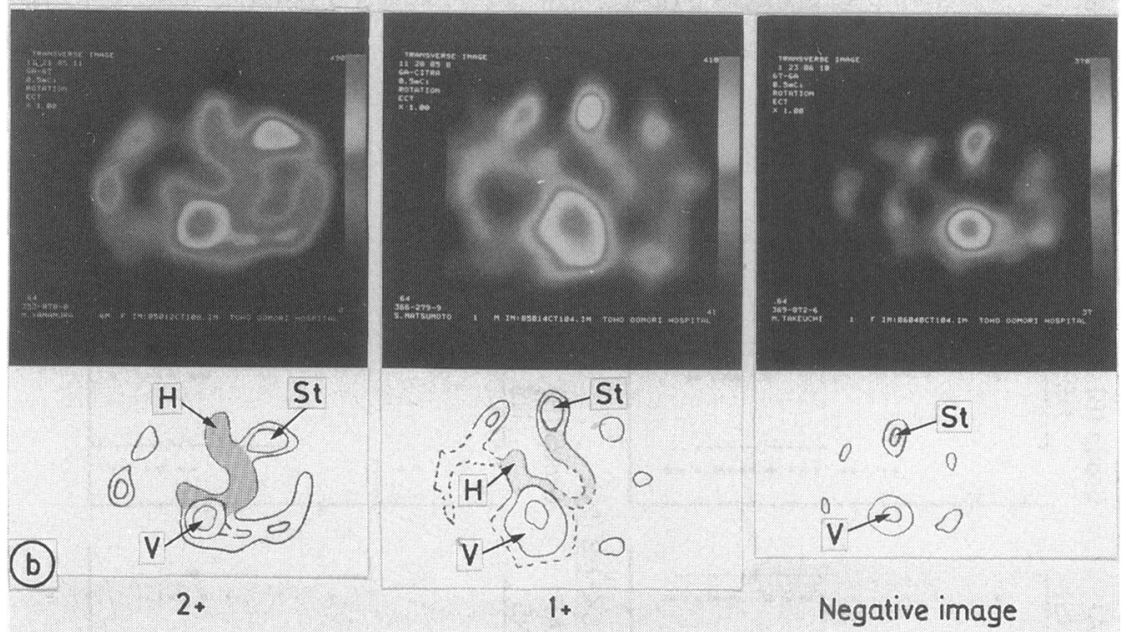

Fig 2 Typical planar and single photon emission computed tomographic (SPECT) images 48 or 72 hours after injection of gallium-67 citrate in patients with myocarditis. (a) The planar images of the positive $1+$ case showed a vague incomplete outline of the heart that was not seen in the negative case. The accumulation of gallium-67 in the heart was equal to that in the spinal bone in the $2+$ case. (b) Typical SPECT images at 48 or 72 hours. No accumulation was seen in the heart of the negative case. The localisation of gallium-67 in the myocardium and not in the ventricular cavities was evident in the $1+$ and $2+$ cases. $H$, heart; $L$, liver; $V$, vertebra; St, sternum.

positive $2+$, showing an obvious silhouette of the heart by planar imaging, or clear identification of the ventricular walls by single photon emission computed tomography imaging; positive $3+$, a greater accumulation of radionuclide in the heart than in the spinal bone.

In the event of disagreement among the three physicians, we used the grade agreed upon by two cardiologists or the mean of the three different grades.

While patients were in hospital the following were measured at least twice a week: complete blood counts, serum total protein and albumin, erythrocyte sedimentation rates, C-reactive protein, and creatine kinase $\mathrm{MB}$ expressed as a fraction of total creatine kinase. The cardiothoracic ratio on chest 

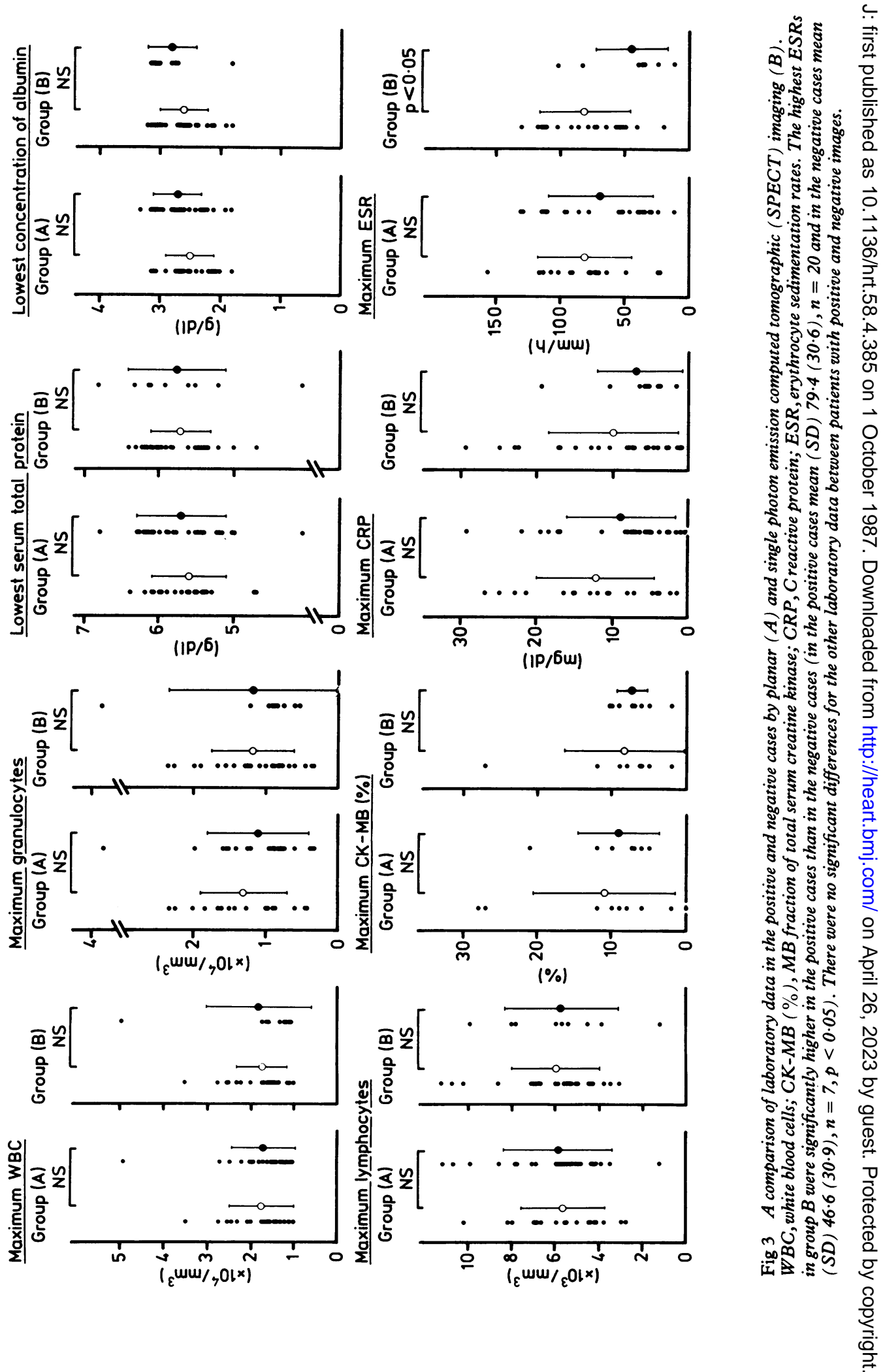
$x$ ray films and the electrocardiograms and echocardiograms were examined at least once a week. When there was an increase or reduction of at least $5 \%$ in the cardiothoracic ratio on the chest $x$ ray films during the clinical course cardiomegaly or the regression of cardiomegaly was diagnosed. Electrocardiograms were analysed by the criteria of Yoshitake et $a l^{4}$ for cardiac rhythm, PQ intervals, corrected QT intervals, significant changes in the voltage of $R$ waves, and ST-T changes. Coronary aneurysms, pericardial effusion, and valve insufficiency were detected by $M$ mode and cross sectional echocardiography and cross sectional colour Doppler echocardiography. We compared these findings and abnormalities in heart sounds (that is distant heart sounds and gallop rhythm) with the results of myocardial imaging with gallium- 67 .

\section{Results}

MYOCARDIAL IMAGING WITH GALLIUM-67

Figure 1 shows both planar and single photon emission computed tomography images in a typical case. At 6 hours there was a remarkable accumulation of gallium-67 in the cardiac silhouette in planar imaging. The single photon emission computed tomography image at 6 hours shows that gallium -67 has accumulated both in the blood in the ventricular cavity and in the myocardium. At 48 hours neither planar nor single photon emission computed tomography imaging showed accumulation of gallium-67 in the heart. The planar image of a $1+$ positive case (fig 2a) shows a vague outline of the heart which was not seen in the negative case. There was a remarkable accumulation of gallium- 67 in the heart in the $2+$ positive case. In positive cases single photon emission computed tomography imaging showed accumulation of the tracer in the myocardium, but not in the blood in the ventricular cavities.

In group $A 19$ patients $(41 \%)$ had positive images (12 cases were $1+$ and 7 cases were $2+$ ) and in group B 25 patients $(64 \%)$ had positive images (15 cases were $1+$ and 10 cases were $2+)$. No patient from either group was graded as $3+$. The frequencies of positive cases in male and female patients were $65 \%$ and $53 \%$ in group $A$ and $80 \%$ and $56 \%$ in group B respectively. There was no significant sex-related difference in the percentages of positive cases. Six positive cases in group B were re-examined at the time of discharge and one case remained positive. A month later this case was negative.

Laboratory data-Figure 3 shows a comparison between positive and negative cases in groups $A$ and $B$. In the acute phase the maximum percentage of the MB fraction of creatine kinase was slightly higher in the positive cases than in the negative cases in both groups but not significantly. In group B the maximum erythrocyte sedimentation rate was significantly higher in the positive cases than in the negative cases (79.4 (30.6) vs $46.6(30.9) \mathrm{mm} / \mathrm{h}, \mathrm{p}<$ $0.05)$. There were no other significant differences in other laboratory data between the positive and negative cases in either group A or B (fig 3).

A COMPARISON OF GALLIUM-67 MYOCARDIAL IMAGING AND CLINICAL FINDINGS IN GROUPS A AND B (TABLES 1 AND 2)

Clinical signs-None of the patients had congestive heart failure. Abnormal heart sounds were noted in $41 \%(19 / 46)$ in group A and 38\% (13/34) in group B. Positive images were obtained in $8(42 \%)$ cases out of the 19 cases with abnormal heart sounds in group A while $9(69 \%)$ cases out of the 13 cases with abnormal heart sounds in group B showed positive images.

Electrocardiograms-Serial electrocardiograms revealed abnormalities in $43 \%(20 / 46)$ patients in group $A$ and $47 \%(16 / 34)$ in group B. Of those cases with abnormal electrocardiograms, $12(60 \%)$ out of 20 showed positive planar images and $13(81 \%)$ out of 16 showed positive single photon emission computed tomography images.

Chest $x$ rays-Only 25 cases in group A and 15 cases in group B had chest $x$ rays that were suitable for reliable assessment of cardiomegaly. In the remain-

Table 1 Correlation of planar images with clinical signs

\begin{tabular}{|c|c|c|c|c|}
\hline \multirow[b]{2}{*}{ Planar images } & \multicolumn{4}{|c|}{ Abnormal findings } \\
\hline & Heart sounds & Cardiomegaly & $E C G$ & Echocardiography \\
\hline Abnormality & $19 / 46(41 \%)$ & $4 / 25(16 \%)$ & $20 / 46(43 \%)$ & $24 / 46(52 \%)$ \\
\hline $\begin{array}{l}\text { Positive 1+ } \\
\text { Positive 2+ } \\
\text { Negative }\end{array}$ & $\begin{array}{r}6 / 19(32 \%) \\
2 / 19(11 \%) \\
11 / 19(57 \%)\end{array}$ & $\begin{array}{l}1 / 4(25 \%) \\
1 / 4(25 \%) \\
2 / 4(50 \%)\end{array}$ & $\begin{array}{l}9 / 20(45 \%) \\
3 / 20(15 \%) \\
8 / 20(40 \%)\end{array}$ & $\begin{array}{r}10 / 24(42 \%) \\
2 / 24(8 \%) \\
12 / 24(50 \%)\end{array}$ \\
\hline
\end{tabular}

Nineteen of these 46 cases had two or more abnormal findings in the above four examinations and 12 cases (63\%) out of the 19 had positive images. 
Table 2 Correlation of single photon emission computed tomography (SPECT) images with clinical signs

\begin{tabular}{llllr}
\hline & \multicolumn{2}{l}{ Abnormal findings } & & \\
\cline { 2 - 5 } SPECT images & Heart sounds & Cardiomegaly & ECG & Echocardiography \\
\hline Abnormality & $13 / 34(38 \%)$ & $2 / 15(13 \%)$ & $16 / 34(47 \%)$ & $18 / 34(53 \%)$ \\
Positive $1+$ & $5 / 13(38 \%)$ & $1 / 2(50 \%)$ & $7 / 16(43 \%)$ & $9 / 18(50 \%)$ \\
Positive 2+ & $4 / 13(31 \%)$ & $0 / 2(0 \%)$ & $6 / 16(38 \%)$ & $4 / 18(22 \%)$ \\
Negative & $4 / 13(31 \%)$ & $1 / 2(50 \%)$ & $3 / 16(19 \%)$ & $5 / 18(28 \%)$ \\
\hline
\end{tabular}

Fifteen of these 34 cases had two or more abnormal findings in the above four examinations and 12 cases $(80 \%)$ out of the 15 had positive images.

der the $x$ rays were unsatisfactory (for example because of a mismatch of the respiratory phase or rotation of the trunk). Cardiomegaly was present in $16 \%(4 / 25)$ in group A and $13 \%(2 / 15)$ in group B, and half the patients in groups $A$ and $B$ showed positive gallium- 67 myocardial images.

Echocardiography-Fifty two per cent $(24 / 46)$ of group A and 53\% (18/34) of group B had abnormal echocardiograms (that is transient pericardial effusion, persistent aneurysms or dilatation of coronary arteries, and transient aortic valve insufficiency). Of these, $50 \%(12 / 24)$ in group $A$ and $72 \%(13 / 18)$ in group B showed positive images by gallium-67 myocardial imaging. The only patient who had transient pericardial effusion, transient aortic valve insufficiency, and persistent coronary aneurysms was classified as positive $(1+)$ by planar imaging; single photon emission computed tomography imaging was not performed in this patient. Five cases in group A, including this case, had aneurysms or appreciable dilatation of coronary arteries at discharge and three $(60 \%)$ of them had positive images. In group B four cases had coronary aneurysms or dilatation and all of them showed positive images.

Cases which had two or more abnormal findings in the above four examinations were said to have clinically suspected myocarditis; in group A 12 $(63 \%)$ out of 19 cases with clinically suspected myocarditis showed positive images while $12(80 \%)$ out of 15 such cases in group B showed positive images.

\section{Discussion}

Infants and children with Kawasaki disease in the acute phase often have abnormal findings such as gallop rhythm and distant heart sounds, prolonged PQ intervals and QTc, as well as echocardiographic evidence of pericardial effusion and valve insufficiency; all of these abnormalities are thought to be due to pancarditis. ${ }^{12}$ Some histopathological studies of specimens obtained by endomyocardial biopsy ${ }^{15}$ have also suggested that the myocardium is often inflamed in the acute phase. Fujiwara and Hamashima classified cardiac lesions into four stages according to the duration of illness at death. ${ }^{6}$ All those who died in the acute phase, especially those in stage 1 (duration of illness to death was 0 to 9 days), showed considerable infiltration of polymorphonuclear leucocytes and lymphocytes into the myocardium. There are also reports of sudden death caused by extension of inflammation to the cardiac conduction systems. ${ }^{7}$ In Kawasaki disease the progression to myocardial fibrosis and hypertrophy from myocarditis is suspected. ${ }^{5}$ Apart from cardiac biopsy conventional investigations are not very helpful in detecting myocarditis and in assessing its severity in patients with abnormal clinical signs. This is mainly because the cardiac symptoms in Kawasaki disease are usually mild and the clinical course is not like that of typical infectious myocarditis. For example changes in electrocardiograms are so mild that only serial tracings reveal faint abnormalities, ${ }^{4}$ and creatine phosphokinase and the MB fraction are increased in only a few cases. ${ }^{8}$

Gallium-67 has a half life of 78 hours and produces four gamma rays with energies suitable for scanning. There is no beta emission, and the radiation dose to the patient is well within the acceptable range. ${ }^{9}$ Recently gallium- 67 citrate imaging was reported to be useful in the detection of clinical and experimental myocarditis, pericarditis, and endocarditis. ${ }^{910}$ Most of the cases reported were the subject of individual case reports and a few workers have reported small series. ${ }^{10}$

The uptake of gallium- 67 by the acute inflammatory lesions is thought to occur in three ways. The most important and best studied one is the role of polymorphonuclear leucocytes. The other two ways are via lymphocytes and the so-called "non-cellular pathway"11 12; both these routes have been proposed as mechanisms of accumulation in states such as agranulocytosis. Gallium-67 resembles the ferric ion in its atomic radius, charges, and in the types of inorganic complexes that it forms; so gallium-67 binds to iron-binding molecules and 
cells-for example lactoferrin, transferrin, ferritin, and siderophores. ${ }^{13}$ The lysosomes of polymorphonuclear leucocytes are rich in lactoferrin and several investigations suggest that lysosomal binding is important in the localisation of gallium- 67 within inflammatory lesions. ${ }^{12-14}$ After accumulating at sites of inflammation leucocytes discharge some of the content of the lysosomes, including lactoferrin. The discharged lactoferrin tends to remain on the receptor sites in tissue macrophages and lymphocytes. ${ }^{13}$

In the acute phase of Kawasaki disease patients show considerable increases in numbers of immature polymorphonuclear leucocytes. The kinetics of lactoferrin have not been studied in patients with Kawasaki disease, but the activity of the enzyme, $\beta$ glucuronidase, released from the lysosomes of polymorphonuclear leucocytes is significantly higher in the acute phase than in the recovery phase. ${ }^{15}$ Characteristic cardiac abnormalities in the acute phase of Kawasaki disease are acute perivasculitis and vasculitis of microvessels and small arteries. Acute pericarditis, interstitial myocarditis, and endocarditis are also seen; the myocarditis is characterised by oedema and infiltrating cells consisting of polymorphonuclear leucocytes and lymphocytes. ${ }^{6}$ At the earliest stage polymorphonuclear leucocytes predominate; and these are rapidly replaced by lymphocytes. ${ }^{16}$ Even biopsy specimens obtained early in the recovery phase contain lymphocytes, plasma cells, histiocytes, and some mast cells rather than polymorphonuclear leucocytes. ${ }^{5}$

These mechanisms for the accumulation of gallium-67 in inflammatory lesions raise the possibility of using gallium-67 citrate to detect myocarditis as a complication of the acute phase of Kawasaki disease. Ito et al and Sty et al reported some positive cases in planar imaging by gallium- 67 citrate in patients with Kawasaki disease, but they did not study the corresponding clinical features in these patients. ${ }^{1718} \mathrm{We}$ believe that polymorphonuclear leucocytes and lactoferrin were responsible for producing the positive images in our series in the acute phase. Lymphocytes or the "noncellular pathway" or both may have been responsible for the positive image at discharge that was found in one patient.

Although the most typical appearance of pericarditis in gallium-67 imaging was a "hot" rim of uneven thickness surrounding the periphery of the entire heart in planar imaging, diffuse, faint activity in the cardiac silhouette has been seen in some cases. ${ }^{19}$ Single photon emission computed tomography imaging was useful because it showed the localisation of gallium-67 in the myocardium as well as in the pericardium of the positive cases. Moreover, this technique showed that the positive images were not the result of high gallium-67 counts in the systemic circulation; this was established by comparing the images at 6 hours with those at 48 or 72 hours in group B. Single photon emission computed tomography imaging was also better than planar imaging; it was more sensitive $(80 \%$ in group $B$ vs $63 \%$ in group A), although this difference was not statistically significant. Single photon emission computed tomography requires more expensive equipment and a longer recording time than planar imaging.

\section{References}

1 Onouchi Z, Tomizawa N, Goto M, Nakata K, Fukuda $\mathbf{M}$, Goto $\mathbf{M}$. Cardiac involvement and prognosis in acute mucocutaneous lymph node syndrome. Chest 1975;68:297-301.

2 Nakano H, Ueda K, Sato A, Tsuchitani Y. Doppler detection of tricuspid regurgitation following Kawasaki disease. Pediatr Radiol 1986;16:123-5.

3 Kawasaki T. Agreement with the guidelines by the Mucocutaneous Lymph Node Syndrome Research Committee [In Japanese]. Nippon Shonikagakkai Zasshi 1984;88:2693.

4 Yoshitake K, Naganuma M, Koike K, et al. Electrocardiographic findings in MCLS. [In Japanese]. Iryo 1980;34:24-7.

5 Yutani C, Go S, Kamiya T, et al. Cardiac biopsy of Kawasaki disease. Arch Pathol Lab Med 1981;105:470-3.

6 Fujiwara H, Hamashima Y. Pathology of the heart in Kawasaki disease. Pediatrics 1978;61:100-7.

7 Fujiwara H, Kawai C, Hamashima Y. Clinicopathologic study of the conduction systems in 10 patients with Kawasaki's disease (mucocutaneous lymph node syndrome). Am Heart J 1978;96:744-50.

8 Yamamoto $S$, Umezawa $T$, Hashiguchi $R$, Saji $T$, Matsuo N. Serum creatine kinase in Kawasaki disease [In Japanese]. Shonika Rinsho 1985;38:2312-6.

9 Reeves WC, Jackson GL, Flickinger FW, et al. Radionuclide imaging of experimental myocarditis. Circulation 1981;63:640-4.

10 Taillefer R, Dionne D. Gallium-67 uptake by the heart. Semin Nucl Med 1983;13:176-8.

11 Merz T, Malmud L, McKusick K, Wagner HN. The mechanism of the Ga-67 association with lymphocytes. Cancer Res 1974;34:2495-9.

12 Dhawan VM, Sziklas JJ, Spencer RP. Localization of Ga-67 in inflammations in the absence of circulating polymorphonuclear leukocytes. J Nucl Med 1978;19:292-4.

13 Hoffer P. Gallium: mechanisms. J Nucl Med 1980;21:282-5.

14 Gelrud LG, Arseneau JC, Milder MS, et al. The kinetics of gallium-67 incorporation into inflammatory lesions: experimental and clinical studies. J Lab Clin Med 1974;63:489-95.

15 Niwa Y, Sohmiya K. Enhanced neutrophilic functions 
in mucocutaneous lymph node syndrome, with special reference to the possible role of increased oxygen intermediate generation in the pathogenesis of coronary thromboarteritis. J Pediatr 1984;104:56-60.

16 Kyogoku K, Fujiyama J, Sawai T. Re-evaluation of the pathological findings in patients with Kawasaki disease (MCLS) [In Japanese]. Shoni Naika 1985;17:731-7.
17 Ito K, Saito C, Nagamatsu K, Hatae Y. Ga-67 scanning in Kawasaki disease (MCLS) [In Japanese]. Kaku Igaku 1981;18:81-6.

18 Sty JR, Chusid MD, Dorrington A. Ga-67 imaging: Kawasaki disease. Clin Nucl Med 1981;6:112-3.

19 Lin DS, Tipton RE. Ga-67 cardiac uptake. Clin Nucl Med 1983;8:603-4. 